\title{
DELTA INFECTION WITHOUT INCREASE IN SEVERITY OF HEPATITIS
}

Sir, - The finding of increased morbidity of HBsAg positive hepatitis with $\delta$ infection by $\mathrm{Dr}$ Smedile and colleagues (Oct. 30, p. 945) is of considerable interest to us because it is contrary to our findings both in 27 parenteral drug abusers with hepatitis B infection who were examined serologically for $\delta$ antigen and antibody and on whom liver biopsies were done and in 41 drug abusers with acute hepatitis B and simultaneous acute $\delta$ infection.

In our group of 27 drug-abusers with hepatitis B infection on whom $\delta$ serology (kindly done by Dr Rizzetto, Turin) and histology were done, 7 had antigenaemia and 1 had anti- $\delta$ antibody. There was no significant difference in the histological findings between $\delta$ positive and $\delta$ negative patients; 4 of the 8 patients with $\delta$ markers had chronic persistent hepatitis and 3 had chronic active hepatitis, while among the $19 \delta$ negative patients 12 had chronic persistent hepatitis and 3 had chronic active hepatitis. Nor were there any differences in the clinical findings. The chronic liver disease found was attributable to non-A, non-B hepatitis in more than $80 \%$ of these patients and it preceded hepatitis B infection in more than $65 \%$.

Of 254 drug-abusers with hepatitis B detected during an epidemic of hepatitis B among them which began in 1980,41 had simultaneous $\&$ infection. ${ }^{11}$ The $\delta$ infection was acute, as indicated by the detection, by enzyme immunoassay, of $\delta$ antigen in the serum during the acute stage of illness only. The proportion with $\delta$ antigenaemia (16\%) was identical to that found by Smedile et al. in their series of fulminant cases. None of our 41 patients with $\delta$ infection have so far shown any increase in severity of illness, such as reported by Smedile et al. There have not been any cases of fulminant hepatitis or death due to hepatitis among our group.

In our study $\delta$ infection did not adversely affect hepatic histological or clinical findings in the initial period of $\delta$ infection. However, our results do not exclude long-term of $\delta$ associated liver disease.

Since all but 1 of our patients with acute $\delta$ infection were non-Italians it may be that ethnic origin is an important factor in the pathogenicity of the $\delta$ agent.

Department of Medical Microbiology,

University College,

Dublin 4, Ireland

A. G. SHATTOCK

J. F. FIELDING

Charitable Infirmary, YVONNE ARTHURS

Dublin

G. D. DOYLE

M. G. Kelly

\footnotetext{
${ }^{1}$ Shattock AG, Kelly MG, Fielding JF, Arthurs Y. Epidemic hepatitis B with delta antigenaemia among Dublin drug abusers. J Irish Medi Assoc 1982; 151: 10

This article is a reproduction of that published in: The Lancet 2(8310), December 1982, p.1286. Pagination may not match that of the original.
} 